\title{
La influencia del factor agroecológico como atributo en la construcción de marca del producto leche
}

The influence of the agroecological factor as an attribute on the brand building of the milk product

Jonnathan Patricio García Garnica. ${ }^{1} \&$ Gabriela Isabel Araujo Ochoa. ${ }^{2}$

Recibido: 20-11-2020 / Revisado: 17-12-2020 /Aceptado: 13-01-2021/ Publicado: 05-02-2021

\begin{abstract}
.
DOI: https://doi.org/10.33262/concienciadigital.v4i1.1.1554
\end{abstract}

Introduction. The Agroecological is a sector little studying within the production system, given that with new technological advances, all processes have been automated, leaving aside the own and natural characteristics of the products. Objective. In the present investigation, it seeks to analyze the agroecological factor as an attribute of the milk product in the construction of the brand of farmers that produce and market agroecological milk in the canton Cuenca. Methodology. The results of the surveys applied to a sample of 384 people from the urban sector of the canton Cuenca of social class C-, D, (lower middle class, low according to (INEC, 2011)). Using data collection techniques in which structured surveys were obtained, obtaining quantitative and qualitative data, tastes and preferences, consumer characteristics were analyzed with multivariable statistical analysis, positioning maps, options and perceptions about ecological and organic. Results. The results show that $12 \%$ do not consume dairy products and $88 \%$ do consume. Additionally, results were obtained in the type of milk they consume, milk brands in the Top of Mind, positioning map in relation to milk brand attributes, and information on agroecological food. Conclusions. This research

1 Universidad Politécnica Salesiana, Carrera de Administración de Empresas, Cuenca, Ecuador. jgarciag2@est.ups.edu.ec, ORCID: https://orcid.org/0000-0001-7892-1668

${ }^{2}$ Universidad Politécnica Salesiana, Carrera de Administración de Empresas, Cuenca, Ecuador. garaujo@ups.edu.ec, ORCID: https://orcid.org/0000-0003-3323-1596 
makes it known that milk producing and marketing companies, which are currently positioned in the city of Cuenca, do not care about being agroecological.

Keywords: Agroecological, Milk, Dairy, Farmers, Positioning, Brands, Attributes.

\section{Resumen.}

Introducción. La agroecología es un sector poco estudiando dentro del sistema de producción, en vista de que, con los Nuevos avances tecnológicos, todos los procesos se han automatizado, dejando a un lado las características propias y naturales de los productos. Objetivo. Analizar el factor agroecológico como atributo del producto de la leche en la construcción de la marca de los ganaderos que producen y comercializan leche agroecológica en el cantón Cuenca. Metodología. Este artículo analiza los resultados de las encuestas aplicadas a una muestra de 384 personas del sector urbano del cantón Cuenca de clase social C-, D, (clase media baja, baja según (INEC, 2011)). Utilizando técnicas de recolección de datos en la cual se hizo encuestas estructuradas obteniendo datos cuantitativos y cualitativos, se analizó los gustos y preferencias, características del consumidor con el análisis estadístico multivariable, mapas de posicionamiento, opciones y percepciones sobre lo ecológico y orgánico. Resultados. Los resultados muestran que el $12 \%$ no consume productos lácteos y el $88 \%$ si consume. Adicionalmente se obtuvo resultados en el tipo de leche que consumen, marcas de leche en el Top of Mind, mapa de posicionamiento con relación a atributos de las marcas de leche, e información sobre el alimento agroecológico. Conclusiones. Con esta investigación se da a conocer que las empresas productoras y comercializadoras de leche, que están posicionadas actualmente en la ciudad de Cuenca no se preocupan de ser agroecológicas.

Palabras claves: Agroecología, Leche, Lácteos, Agricultores, Posicionamiento, Marcas, Atributos.

\section{Introducción.}

Rodríguez (2014), alega que la agroecología es una ciencia y una serie de prácticas tan antiguas como los orígenes de la agricultura, donde convergen dos disciplinas científicas: la agronomía y la ecología, además Altieri (1999) incorpora el concepto de la agricultura no sólo en la producción sino también en la sostenibilidad ecológica del sistema de producción, a sabiendas que la leche es un alimento muy importante para las personas brindando varios beneficios para la salud, ayudando a progresar, formar, y fortificar los huesos de las personas, también que una de las ventaja de la leche es que tiene proteínas Tasat (2017), las cuales nos ayudan para la alimentación sana MANU (2016), además en nuevas investigaciones se incorporan nuevos enfoques de la agricultura ligado al medio ambiente Restrepo M, Angel S, \& Prager M (2000). 
La presente investigación parte de las necesidades que tienen los ganaderos que producen y comercializan leche agroecológica en el cantón Cuenca.

En esta investigación se da a conocer:(1) los atributos que relacionan los clientes con la leche agroecológica, (2) se conoce a la mejor empresa posicionada de lácteos en la ciudad de Cuenca, (3) los atributos que relacionan el cliente con la empresa de lácteos y (4) motivos de porque consumirían productos agroecológicos y cuanto están dispuestos a pagar por la leche agroecológica.

En la actualidad las personas buscan nuevas formas de cuidar el medio ambiente, utilizando diferentes procesos para mejorar sus productos, que sean sanos y sin químicos, es por ello por lo que (MAGAP, 2014), con el tema Agroecología impulsa la mejora de los productos dando a conocer que:

La Agroecología se reconoce como el modelo agrario del futuro. Miles de comunidades campesinas y agricultores del Ecuador y del mundo lo impulsan como respuesta a la crisis agroalimentaria que afronta el mundo. De igual manera el MAGAP (2014), también impulsa a la agroecología dando a conocer lo que destaca Clara Nicholls, presidenta de La Sociedad Científica Latinoamericana (SOCLA) que, "la clave para el desarrollo de la agricultura familiar es que la tierra cumpla con una función social, lo que implica que sea productiva y sea trabajada por los agricultores familiares, porque en sus manos está la conservación de los recursos fitogenéticos.

En las partes rurales del cantón Cuenca, los ganaderos no conocen acerca de los precios que estipula la ley para sus productos o si los conoce no pueden vender a un precio justo, esta situación puede ser dada por diferentes razones, (1) Que existe mucha competencia, (2) La venta a intermediarios. (3) No exista compradores para sus productos, ante esta circunstancia el MAGAP (2013) da a conocer a sus diferentes asociaciones que el Acuerdo Ministerial No. 394. Para garantizar un pago justo a los productores de leche donde;

El precio por litro de leche al productor está indexado en un 52.4\% al precio de venta al público de la leche UHT en funda en el mercado nacional más componentes como: calidad higiénica y calidad sanitaria. El precio de venta al público de la leche UHT en funda es de 80 centavos de dólar; razón por la cual, el precio de indexación por litro de leche pagado al productor es de 42 centavos de dólar más bonificaciones por calidad.

Para complementar esta idea del producto agroecológico, se debería tener en claro el significado de la marca Mestres (2012). Las marcas trasmiten diferentes significados, nombre, expresión, distintivo o diseño para que el cliente pueda reconocer facialmente la marca, por lo que (Batey, 2013) y (KOTLER, 2012) considera que el significado de la marca media entre los productos y las motivaciones de los consumidores, y de ese modo determina el comportamiento del consumidor. Kotler \& Armstrong (2007) plantean sus estrategias de 
posicionamiento, mismos que muestran a los consumidores las percepciones de sus marcas contra los productos de la competencia en dimensiones de compra importantes.

\section{Metodología.}

Para la presente investigación se utilizó métodos concluyentes descriptivos con muestra simple. Se realizó una investigación bibliográfica documental, para establecer las teorías más relevantes y actualizadas para el desarrollo del estado del arte, referente a atributos de marca. También se utilizó la técnicas de recolección de datos en la cual se hizo encuestas estructuradas obteniendo datos cuantitativos y cualitativos, se analizó los gustos y preferencias, atributos de marca, comportamiento de compra, características del consumidor con el análisis estadístico multivariable, opciones y percepciones sobre lo ecológico y orgánico, mediante las técnicas escalamiento multidimensional (EMD) expuesto por (Malhotra, 2008), análisis discriminante, análisis factoriales y análisis componentes principales expuestos por pedro (Castejón , Lechuga, \& Martínez, 2015) con esto se puedo lograr Determinar el posicionamiento relativo de las principales marcas de leche en la ciudad de Cuenca y Analizar la influencia de los factores ecológicos y orgánicos como atributos de marca el consumo de leche procesado en la ciudad de Cuenca.

Para este caso se aplicó una investigación cuantitativa con el fin de conocer los atributos del producto leche en la construcción de marca.

Al ser la leche un producto de primera necesidad, esta es consumida y adquirida sin distinción de edad, ni sexo, por lo cual, por operatividad de la presente investigacion se tomo a la población de estudio, la poblacion urbana del canton Cuenca de clases sociales C- y D (clase media baja , baja) según (INEC, 2011), la tabla 1.

\begin{tabular}{lr}
\hline \multicolumn{2}{c}{ Nivel Socioeconómico } \\
\hline Clase social ALTA (A) & $1,90 \%$ \\
Clase social MEDIA(B) & $11,20 \%$ \\
Clase social MEDIA ALTA (C+) & $22,80 \%$ \\
Clase social MEDIA BAJA (C-) & $49,30 \%$ \\
Clase social BAJA (D) & $14,90 \%$ \\
\hline
\end{tabular}

Tabla 1: Nivel socio económico

Fuente: (INEC, 2011)

Para poder desarrollar el cálculo de la muestra se tiene una población total en Cuenca: 614,539.00 (INEC, 2019) esto se multiplica la Población de Cuenca que es $614.539,00$ por el $65 \%$ de personas del sector urbano nos muestra el resultado de 399.451,00 habitantes (hombres y mujeres del sector urbano de la ciudad de Cuenca),del total de Sector urbano que es $399.451,00$ la cual se multiplica por $64.2 \%$ esto es la sumatoria de la clase C- y D (clase 
media baja, baja) que está en la tabla anteriormente expuesta, multiplicado la población urbana con la clase social nos da un total de 256.448,00 personas.

Después se desarrolla el cálculo de la muestra teniendo la siguiente información, una población de 25.448,00 del sector urbano de la ciudad de Cuenca de clase social C-y D (INEC, 2011), con un nivel de confianza de 1.96, con la proporción esperada de éxito de 0.5 y el margen de error con 0.5 obteniendo una muestra de 384 encuestas que fueron aplicadas a los habitantes del sector urbano de la ciudad de Cuenca

\section{Resultados.}

Las respuestas de las encuestas tabuladas estadísticamente se realizó mediante en el Software Statistical Package for the Social Sciences (SPSS),la información demográfica de los 384 habitantes de la poblacion urbana del cantón Cuenca de clases sociales C-y D, (clase media baja, baja) fue : el $59 \%$ encuestados de género femenino y el $41 \%$ pertenece al género masculino; se analizó el estado civil de las personas encuestadas, de las cuales al alrededor de $34 \%$ son personas casados(a), el 47\% solteras(a),el 7\% divorciados(a), el $9 \%$ unión libre y el 3\% viudo(a); de la misma forma se trabajó con rangos de edades, para poder diferenciar los gustos y preferencias, donde la mayoría de las personas pertenecen al rango de 18 a 28 años con el $41 \%$, el $31 \%$ pertenece de 28 a 38 años las otras partes pertenecen al $9 \%, 11 \%$, $5 \%, 1 \%, 2 \%$ de 38 años en adelante.

\section{A. Consumo de lácteos y motivos por los cuales no consumen lácteos por sexo, edades.}

De los datos obtenidos de la estadística descriptivo de los encuestados se puede determinar que el $88 \%$ de las personas consumen lácteos, dentro de los que consumen lácteos el 59\% son del sexo femenino y el $41 \%$ masculino, mientras que el $12 \%$ no acostumbran a consumir lácteos de los cuales el $64 \%$ es de sexo femenino y $36 \%$ masculino.

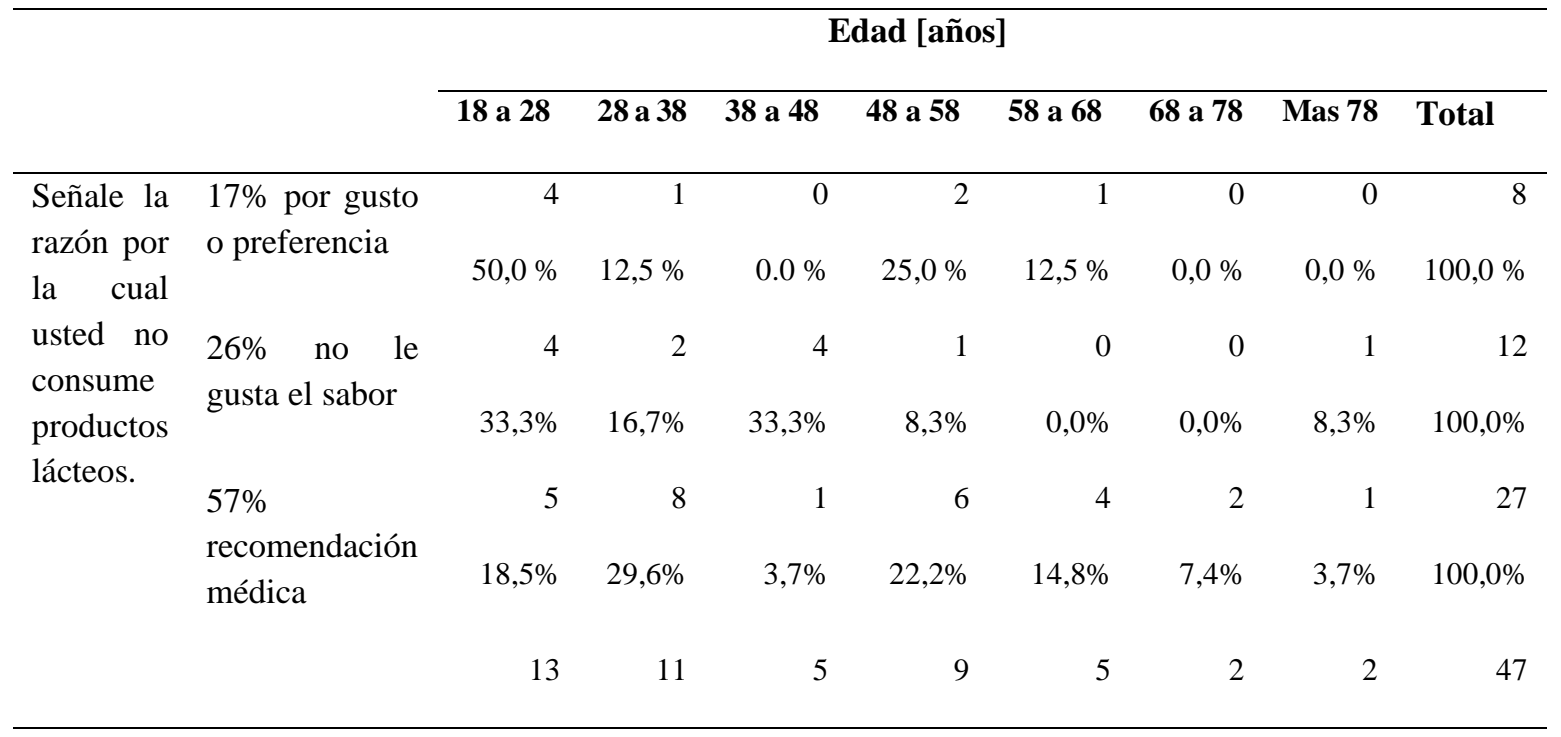




\begin{tabular}{lllllllll}
\hline Total & $27,7 \%$ & $23,4 \%$ & $10,6 \%$ & $19,1 \%$ & $10,6 \%$ & $4,3 \%$ & $4,3 \%$ & $100 \%$
\end{tabular}

Tabla 2: Edad frente al no consumo de productos lácteos.

Fuente: Elaboración propia

Las personas encuestadas que respondieron acerca de la razón por la que no consumen productos lácteos, se hallan en un rango de edad comprendida entre los 18 hasta los 68 años de edad, cuyos resultados son los siguientes: 1) radica principalmente por recomendación médica representando el $57 \%$; 2) el $26 \%$ no consume lácteos debido a que no le gusta el sabor; 3) y el $17 \%$ no consume productos lácteos por gusto o preferencia, ya que en la actualidad existe mayor número de personas que prefieren ser veganos.

B. Tipos de lácteos que consume la población urbana de la ciudad Cuenca de clase social $C$ - y $D$.

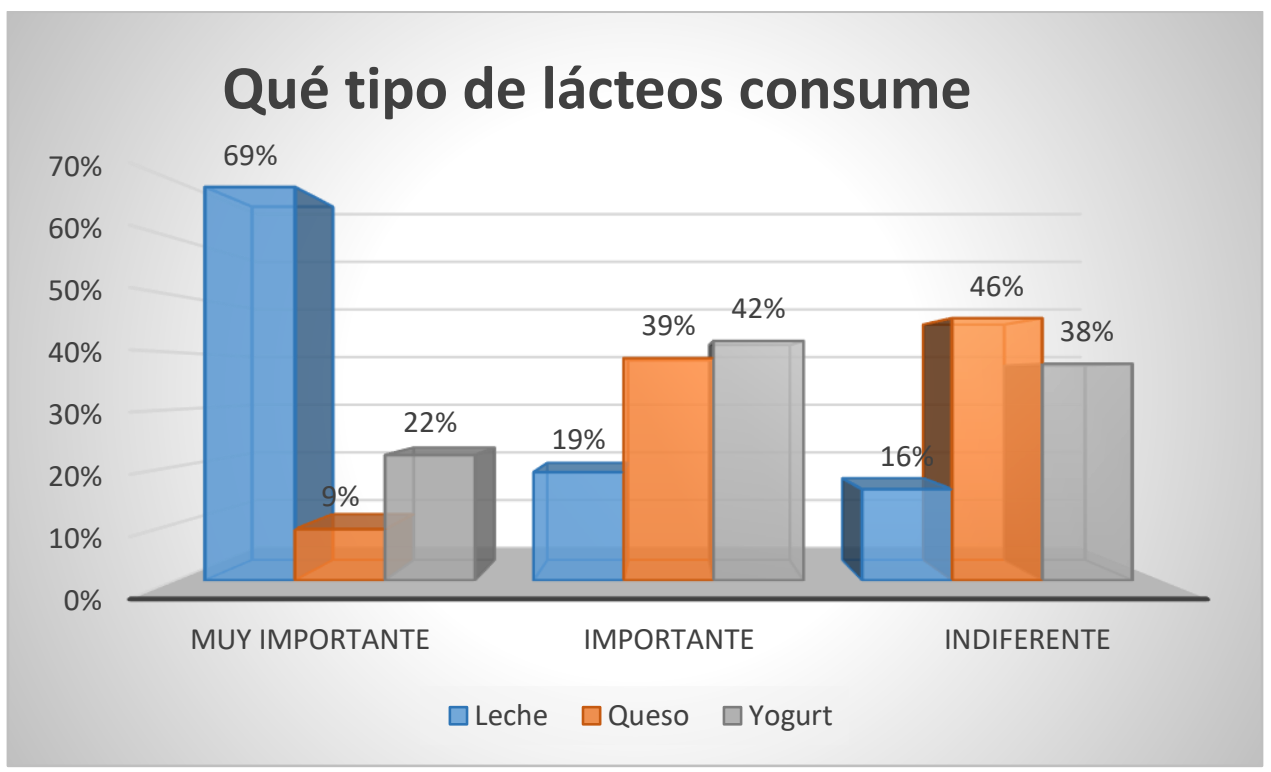

Figura 1: Tipos de lácteos que consumen.

Fuente: Elaboración propia.

En una dieta diaria, en la población de la ciudad de Cuenca, se observó que el 69\% de los habitantes consumen leche y considera que es muy importante este lácteo, por los múltiples benéficos que brinda a las personas.

Como se puede apreciar en la figura 1, el $42 \%$ de la población considera que el yogurt es importante para el consumo en las familias y el $46 \%$ de la población considera que el queso es indiferente porque contiene grasas saturas y alto contenido de sal.

Dando a conocer la mayor frecuencia de compra de leche es semanal con el $44.8 \%$, el $39.17 \%$ la frecuencia de compra es diario y el $16.02 \%$ es mensual. 
C. Edades frente a los tipos de leche que consumen población urbana de la ciudad Cuenca de clase social $C$ - y D.

Por diferentes motivos, sean estos de salud, cuidado personal, gustos, las empresas de lácteos han creído conveniente la adaptación de variedades de presentaciones de la leche, obteniendo de esta manera la siguiente aceptación de la población

La mayoría de las personas que consumen leche prefieren que sea semidescremada con el $22 \%$, el $21 \%$ prefieren entera, el $15 \%$ descremada, $14 \%$ cruda, $9 \%$ deslactosada, el $8 \%$ en polvo, el $7 \%$ fortificada, $4 \%$ vegetariana.

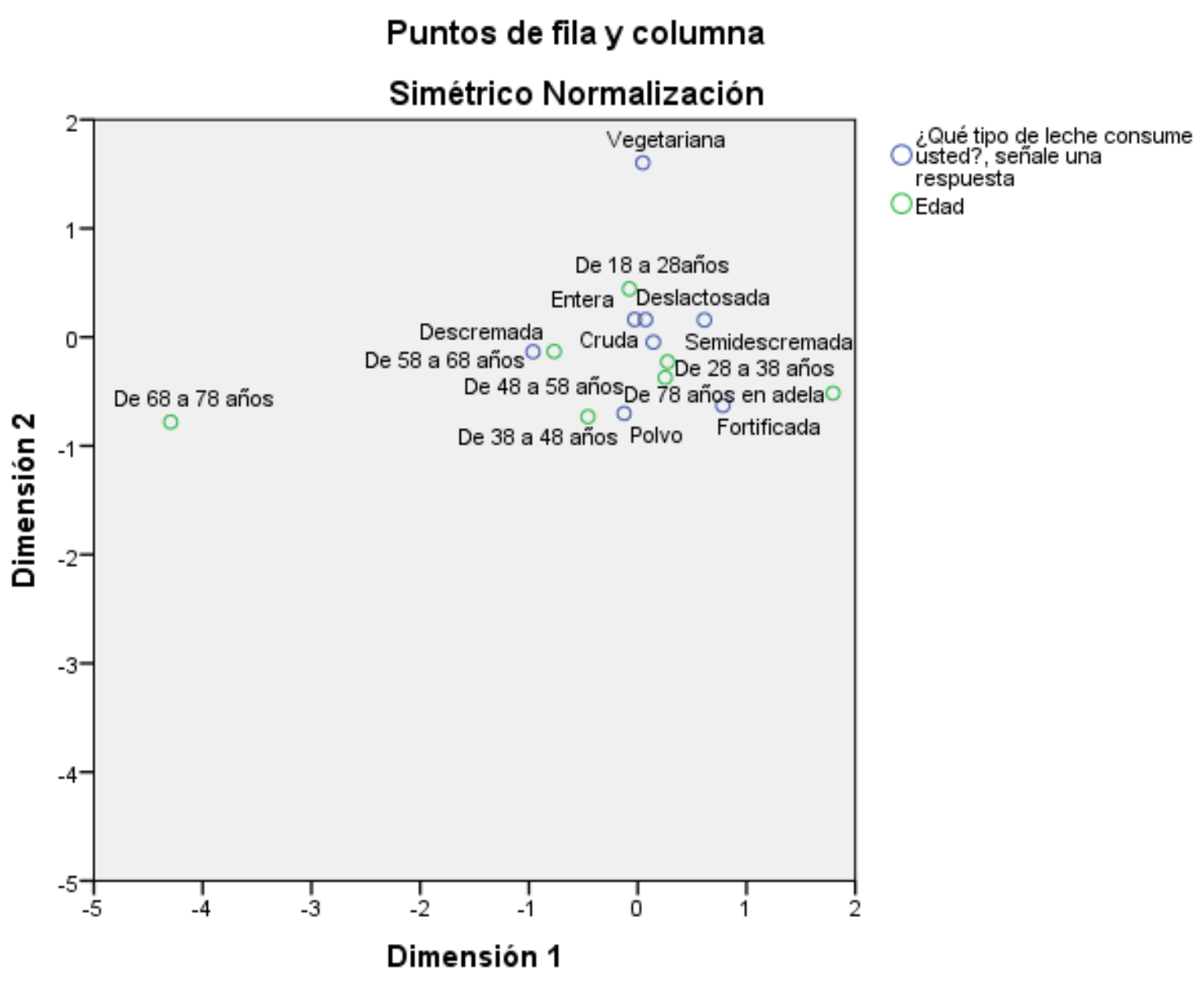

Figura 2. Tipo de leche que consume con respecto a edades.

Fuente: Elaboración propia.

En la figura 2 de análisis de correspondencia muestra que las personas que están en el rango de 18 a 28 años consumen leche entera, deslactosada y vegetariana, por motivo de nutrición u otros, de 28 a 38 años consume leche semidescremada y cruda, de 38 a 58 años consumen 
ISSN: 2600-5859

Vol. 4, N¹.1, p. 198-212, Febrero, 2021

leche en polvo, de 58 a 68 años consumen leche descremada y de 78 años en adelante consumen leche fortificada por el motivo que fortalecen su salud.

D. El posicionamiento relativo de las principales marcas de leche en la ciudad de Cuenca.

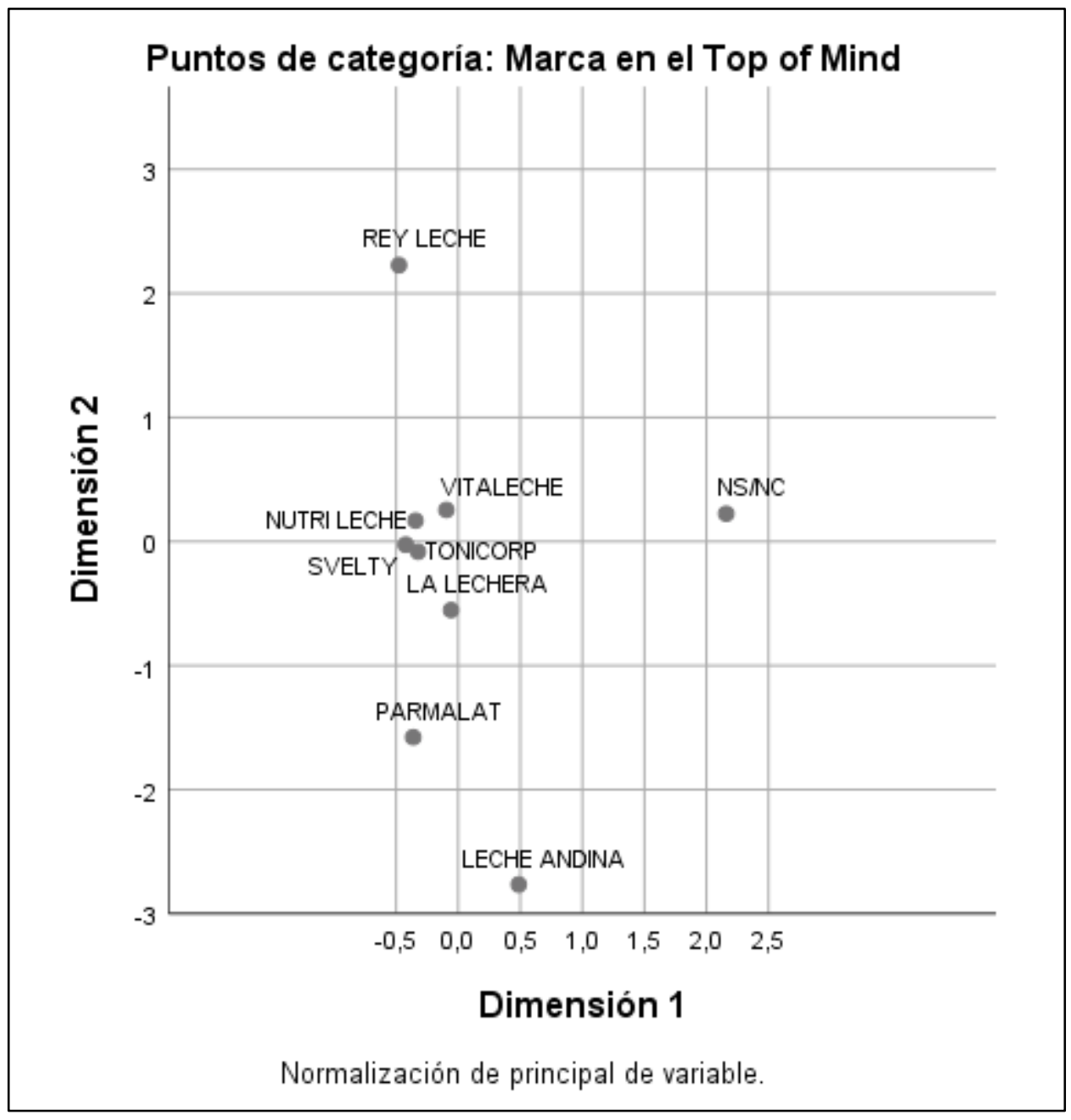

Figura 3: Marcas en el Top of Mind.

Fuente: Elaboración propia de la base de la base de datos (SPSS)

Para poder estar en el top debe ser recordada fácilmente la marca, en la figura 3 muestra a la mejor empresa posicionada, como resultados de esta figura se toma en cuenta a las marcas que se encuentran más cercanas al punto cero del plano cartesiano; estas poseen mayor posicionamiento debito que son recordadas fácilmente por el cliente; y las que están más alejadas al punto cero son las que no se encuentran con un posicionamiento significativo, esto se debe a que la gente no le reconoce fácilmente por sus atributos o tal vez son marcas nuevas en el mercado. 
En la figura 3 conocemos el lugar de donde está posicionada cada empresa de leche de la ciudad de Cuenca. En el puesto número uno la mejor marca posicionada en la ciudad de

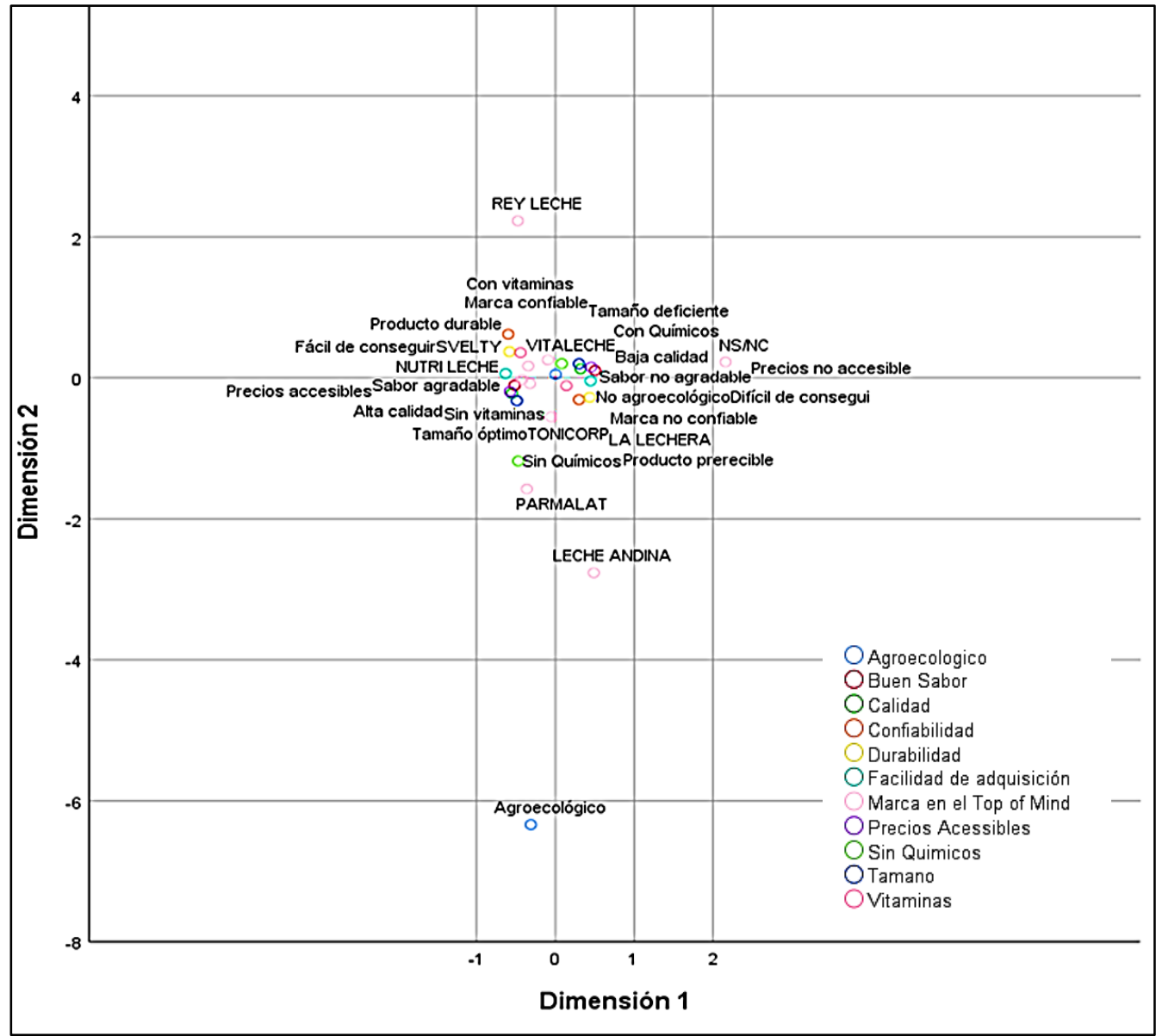

Cuenca es NUTRI LECHE, en el segundo puesto está SVELTY, en el tercer puesto es la marca VITALECHE, en el cuarto lugar es la marca TONICORP, en el quinto lugar es la marca LECHERA, en el sexto lugar la es marca PARMALAT, en el séptimo puesto se tiene a la marca REY LECHE y como octava marca se tiene a LECHE ANDINA

Figura 4: Mapa de posicionamiento con relación a atributos

Fuente: Elaboración propia de la base de datos (SPSS)

En el anterior mapa de posicionamiento ya nos dio cual es la mejor empresa posicionada de leche en la ciudad de Cuenca. En este mapa de posicionamiento de multivariables nos da a conocer también a la mejor empresa posicionada y los atributos que relaciona el cliente con la marca, en el cual la mejor empresa posicionada de leche es la marca NUTRÍ LECHE con los siguientes atributos: fácil de conseguir, precios accesibles, sabor agradable y alta calidad. La segunda empresa posicionada de leche es la marca SVELTY, en donde el cliente la relaciona con los siguientes atributos: producto durable, marca confiable, con vitaminas. La tercera empresa posicionada es VITALECHE con los siguientes atributos: marca confiable, con vitaminas. 
En cuanto al mapa de posicionamiento (Figura 4), es notoria la falta de relación de la agroecología con una marca de leche, tal vez debido a la alta industrialización de esta. Cabe recalcar que algunos ganaderos del cantón ya son asociados como productores agroecológicos, pero al no ser conocidos pierden participación en el mercado cuencano.

E. Análisis de la influencia de los factores ecológicos y orgánicos como atributos de marca y el consumo de leche procesado en la ciudad de Cuenca.

Como ya se conoce a la mejor empresa posicionada la cual es NUTRI LECHE y sus atributos favorables son: fácil de conseguir, precios accesibles, producto durable, marca confiable, sabor agradable y con vitaminas.

Además el resultado es que ninguna empresa que vende leche en la ciudad de Cuenca no se preocupa por ser agroecológica, por lo tanto se procedió a realizar las encuestas estructuradas con el termino agroecológico, donde la gran parte de las personas encuestadas relacionan la agroecológico con salud el $29 \%$, el $24 \%$ relacionan con agricultura responsable, el $15 \%$ con medio ambiente, el $9 \%$ con industria, el $7 \%$ con innovación, $6 \%$ con solidaridad, el $5 \%$ con costo , el $4 \%$ con procesado y el $1 \%$ con artificial.

\begin{tabular}{|c|c|c|c|c|c|c|c|c|c|c|c|c|}
\hline & \multicolumn{10}{|c|}{$\begin{array}{c}\text { ¿Si existiera una leche que prometiera ser agroecológica } \\
\text { que posibilidad de consumo usted le daría? }\end{array}$} & \multirow[b]{2}{*}{ Total } \\
\hline & & $10 \%$ & $20 \%$ & $30 \%$ & $40 \%$ & $50 \%$ & $60 \%$ & $70 \%$ & $80 \%$ & $90 \%$ & $100 \%$ & \\
\hline \multirow{10}{*}{$\begin{array}{c}\text { ¿Qué tan } \\
\text { importante } \\
\text { considera } \\
\text { usted que un } \\
\text { alimento sea } \\
\text { agroecológico? }\end{array}$} & \multirow{2}{*}{$\begin{array}{l}\text { Muy } \\
\text { Importante }\end{array}$} & 1 & 1 & 4 & 6 & 20 & 6 & 11 & 26 & 28 & 51 & 154 \\
\hline & & $0,6 \%$ & $0,6 \%$ & $2,6 \%$ & $3,9 \%$ & $13,0 \%$ & $3,9 \%$ & $7,1 \%$ & $16,9 \%$ & $18,2 \%$ & $33,1 \%$ & $100,0 \%$ \\
\hline & \multirow[t]{2}{*}{ Importante } & 2 & 2 & 5 & 12 & 40 & 15 & 14 & 20 & 21 & 16 & 147 \\
\hline & & $1,4 \%$ & $1,4 \%$ & $3,4 \%$ & $8,2 \%$ & $27,2 \%$ & $10,2 \%$ & $9,5 \%$ & $13,6 \%$ & $14,3 \%$ & $10,9 \%$ & $100,0 \%$ \\
\hline & \multirow[t]{2}{*}{ Indiferente } & 1 & 4 & 2 & 1 & 5 & 2 & 4 & 3 & 1 & 3 & 26 \\
\hline & & $3,8 \%$ & $15,4 \%$ & $7,7 \%$ & $3,8 \%$ & $19,2 \%$ & $7,7 \%$ & $15,4 \%$ & $11,5 \%$ & $3,8 \%$ & $11,5 \%$ & $100,0 \%$ \\
\hline & \multirow{2}{*}{$\begin{array}{l}\text { Poco } \\
\text { Importante }\end{array}$} & 0 & 0 & 1 & 2 & 1 & 0 & 0 & 0 & 2 & 2 & 8 \\
\hline & & $0,0 \%$ & $0,0 \%$ & $12,5 \%$ & $25,0 \%$ & $12,5 \%$ & $0,0 \%$ & $0,0 \%$ & $0,0 \%$ & $25,0 \%$ & $25,0 \%$ & $100,0 \%$ \\
\hline & \multirow{2}{*}{$\begin{array}{l}\text { Nada } \\
\text { Importante }\end{array}$} & 0 & 0 & 0 & 0 & 1 & 0 & 0 & 0 & 1 & 0 & 2 \\
\hline & & $0,0 \%$ & $0,0 \%$ & $0,0 \%$ & $0,0 \%$ & $50,0 \%$ & $0,0 \%$ & $0,0 \%$ & $0,0 \%$ & $50,0 \%$ & $0,0 \%$ & $100,0 \%$ \\
\hline \multirow[t]{2}{*}{ Total } & & 4 & 7 & 12 & 21 & 67 & 23 & 29 & 49 & 53 & 72 & 337 \\
\hline & & $1,2 \%$ & $2,1 \%$ & $3,6 \%$ & $6,2 \%$ & $19,9 \%$ & $6,8 \%$ & $8,6 \%$ & $14,5 \%$ & $15,7 \%$ & $21,4 \%$ & $100,0 \%$ \\
\hline
\end{tabular}

Tabla 3: Importancia para el consumo de la leche agroecológico Fuente: Elaboración propia de la base de datos (SPSS).

Actualmente todas las personas están procupados por tener productos sanos y sin químicos, que no destruyan a la persona y así mantenerse saludables todos los dias, principalmente las personas que se preocupan por tener los productos más saludables y de alta calidad son 
agriculotres y ganaderos, ellos prometen a la población un nuevo producto agroecológico, es por eso que la tabla 3 muestra que las personas encuestadas consideran el término agroecologico como muy importante porque si existiera una leche que prometiera ser agroecológica la posibilidad de consumo que dieron es el 100\%; las personas que respondieron las encuestas también manifestaron los motivos por lo que consumieran un producto agroecológico, siendo los más relevantes, por salud, agricultura responsable y el medio ambiente.

\begin{tabular}{|c|c|c|c|c|}
\hline & & $\begin{array}{r}\text { ¿Conoce a los a } \\
\text { comercializan lec }\end{array}$ & $\begin{array}{l}\text { res que } \\
\text { cológica? }\end{array}$ & \\
\hline & & SI & NO & Total \\
\hline & $\mathrm{Si}$ & 9 & 6 & 15 \\
\hline ¿Ha consumido & & $60,0 \%$ & $40,0 \%$ & $100,0 \%$ \\
\hline lácteos & No & 6 & 315 & 321 \\
\hline & & $1,9 \%$ & $98,1 \%$ & $100,0 \%$ \\
\hline Total & & 15 & 321 & 336 \\
\hline & & $4,5 \%$ & $95,5 \%$ & $100,0 \%$ \\
\hline
\end{tabular}

Tabla 4: Ganaderos que comercializan leche agroecológica vs consumo

Fuente: Elaboración propia de la base de datos (SPSS).

En la actualidad hay asociaciones de ganaderos y agricultores que promueven impulsar el desarrollo y fortalecimiento de la producción para tener precios justos y productos sin químicos.

En las zonas rurales se encuentran ubicados estas organizaciones las cuales no son reconocidas, no pueden vender sus productos y tienen dificultades de traslado de sus productos a otros mercados, donde los ganaderos y agricultores utilizan productos orgánicos y procesos más sacrificados, para ofrecer al público productos de alta calidad y sanos, es por ello que, en la tabla 4 muestra que el $95.5 \%$ de la población no ha consumido lácteos agroecológicos y tampoco conoce a los ganaderos que comercializan leche agroecológica; mientras que tan solo el $5 \%$ consume y conoce a los ganaderos que comercializan la leche agroecológica.

¿Cuánto pagaría usted por un litro de leche agroecológica?

\begin{tabular}{ccc}
\hline Costo & Frecuencia & Porcentaje válido \\
[USD] & {$[\%]$} \\
\hline
\end{tabular}




\begin{tabular}{lcc}
\hline $\begin{array}{ll}\text { Menos de } 0.80 \mathrm{ctv} \\
0.80-0.90 \mathrm{cvt}\end{array}$ & 84 & 25 \\
$1.00-1.10$ & 114 & 16 \\
$1.10-1.20$ & 54 & 3 \\
Total & 10 & 100 \\
Perdidos & 337 & \\
Total & 47 & \\
Tabla 5: Disponibilidad de pago por leche agroecológica \\
Fuente: Elaboración propia de la base de datos (SPSS).
\end{tabular}

Para garantizar el pago justo a los productores de leche, MAGAP (2013) da a conocer a las diferentes organizaciones el "Acuerdo Ministerial No. 394 el precio de indexación por litro de leche pagado al productor es de 42 centavos". cuyo objetivo es mejor el pago de comprador a ganadero y la venta no sea menor a este precio. Es por ello que la población cuencana que no consumían y no conocían a los ganaderos que comercializan leche agroecología y sabiendo el gran sacrificio que hacen para mejorar el producto (sanos y sin químicos), están dispuestos a pagar de 0.80ctv. a 0.90ctv. el litro de leche al ganadero.

\section{Conclusiones.}

- En el cantón Cuenca, el 88\% de sus habitantes consumen lácteos, siendo un mercado potencial para esta industria. A pesar de que el 12\% restante, no consume lácteos, esto no representa una amenaza para la toma de decisiones para los atributos de la leche agroecológica, debido a que la mayoría son casos de recomendación medica

- El producto lácteo más consumido en el cantón Cuenca es la leche, considerada como un alimento muy importante para el consumo de sus hogares. Tomando en cuenta al mercado cuencano, la edad que más consume leche es la comprendida entre los 18 a 28 años, los cuales prefieren consumir leche entera, deslactosada y vegetariana; de la misma forma la población comprendida entre los 28 a 38 años consumen leche semidescremada y cruda, de 38 a 58 años consumen leche en polvo, de 58 a 68 años consumen leche descremada y de 78 años en adelante consumen leche fortificada. Esto debido a las diferentes necesidades que cada tipo de leche puede satisfacer.

- En el cantón Cuenca, existe varias empresas dedicadas a la producción y comercialización de leche, y según la investigación realizada la empresa posicionada en el mercado es la marca NUTRILECHE con los siguientes atributos: fácil de conseguir, precios accesibles, producto durable, marca confiable, con vitaminas, sabor agradable. 
- Dentro de las empresas de leche en la ciudad de Cuenca, ninguna de estas se preocupa de ser agroecológica, por el reconocimiento y aceptación que tienen en el mercado en la actualidad.

- En la actualidad la agroecología está buscando mejorar sus productos (sanos y sin utilizar químicos) es por ello, que la mayoría de las personas relacionan el término agroecológico, con salud, agricultura responsable y medio ambiente, también consideran que si existiera una leche que prometiera ser agroecológica la posibilidad de consumo sería del $100 \%$.

- Al darse cuenta de la gran importancia de los productos agroecológicos, la población está dispuesta a pagar por un litro de leche agroecológica de $0.80 \mathrm{ctv}$. a $0.90 \mathrm{ctv}$.

- Para lograr posicionar a una nueva marca de leche, en este sentido a la agroecológica, se debe además tomar en cuenta los atributos de la marca actual mayor posicionada, los cuales son: fácil de conseguir, precios accesibles, sabor agradable y alta calidad.

- A pesar de que la agroecología, beneficia a la salud de las personas, la misma no es muy conocida, y de esto se puede desprender, futuras investigaciones, en el sentido de ¿por qué ninguna empresa se preocupa de tener leche agroecológica?, y además el ¿por qué no impulsar a los ganaderos que producen esta leche?

\section{Referencias bibliográficas.}

Kotler, p., \& Armstrong, G. (2007). Marketing. México: Pearson edcación.

Altieri, M. (1999). AGROECOLOGIA Bases científicas para una agricultura sustentable. peru: Nordan cominidad .

Batey, M. (2013). El significado de la marca. Buenos Aires: GRANICA.

Castejón , P. J., Lechuga, M. L., \& Martínez, Ú. F. (2015). Guía Práctica de Estadística aplicada a la empresa y al marketing. Madrd: Paraninfo.

INEC. (Diciembre de 2011). Instituto Nacional de Estadística y Censos. Recuperado el 9 de Noviembre de 2019, de Instituto Nacional de Estadística y Censos: https://www.ecuadorencifras.gob.ec/documentos/webinec/Estadisticas_Sociales/Encuesta_Estratificacion_Nivel_Socioeconomico/11122 0_NSE_Presentacion.pdf

INEC. (2019). Instituto Nacional de Estadísticas y Censos. Recuperado el $10 \mathrm{de}$ OCTUBRE de 2019, de Instituto Nacional de Estadísticas y Censos.

KOTLER, P. (2012). Dirección de marketing . Mexico: Pearson.

MAGAP. (4 de Septiembre de 2013). Ministerio de Agricultura, Ganadería, Acuacultura y Pesca. Recuperado el 20 de Noviembre de 2019, de Ministerio de Agricultura, Ganadería, Acuacultura y Pesca: https://www.agricultura.gob.ec/acuerdoministerial-no-394/ 
MAGAP. (Julio de 2014). Ministerio de Agricultura y Ganaderia. Recuperado el 07 de Noviembre de 2019, de Ministerio de Agricultura y Ganaderia: https://www.agricultura.gob.ec/en-el-magap-se-analizo-la-agroecologia-comolanueva-matriz-agro-alimentaria/

Malhotra, N. (2008). Investigacion de Mercados. Mexico: Pearson Educación.

MANU, B. (17 de Noviembre de 2016). THE GOURMET JOURNAL. Recuperado el 18 de Noviembre de 2019, de THE GOURMET JOURNAL:

https://www.thegourmetjournal.com/a-fondo/beneficios-leche-de-vaca/

Mestre, M. S. (2012). Marketing conceptos y estrategias. Madrid: Piramide.

Restrepo M, J., Angel S, D., \& Prager M, M. (Septiembre de 2000). Actualización Profesional en Manejo de Recursos. Recuperado el 19 de septiembre de 2019, de Agroecología:

http://www.fao.org/fileadmin/user_upload/training_material/docs/Agroecologia.pdf

Rodríguez, R. (2014). La agroecología está presente Mapeo de productores agroecológicos y del estado de la agroecología en la sierra y costa. Recuperado el 19 de Septiembre de 2019, de Una publicación de Heifer - Ecuador: http://www.heifer-ecuador.org/wpcontent/uploads/2015/01/1_La_agroecologia_esta_presente_ES.pdf

Tasat, S. (10 de Julio de 2017). NUTRIGLAM. Recuperado el 15 de Noviembre de 2019, de NUTRIGLAM: https://www.infobae.com/tendencias/nutriglam/2017/07/10/lechede-vaca-7-beneficios-de-la-madre-de-los-lacteos/

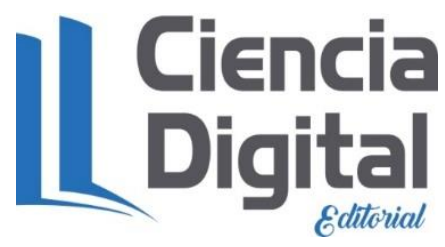




\section{PARA CITAR EL ARTÍCULO INDEXADO.}

García Garnica, J. P., \& Araujo Ochoa, G. I. (2021). La influencia del factor agroecológico como atributo en la construcción de marca del producto leche . ConcienciaDigital, 4(1.1), 198-212. https://doi.org/10.33262/concienciadigital.v4i1.1.1554

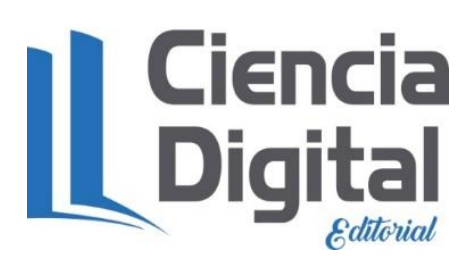

El artículo que se publica es de exclusiva responsabilidad de los autores y no necesariamente reflejan el pensamiento de la Revista Conciencia Digital.

El artículo queda en propiedad de la revista y, por tanto, su publicación parcial y/o total en otro medio tiene que ser autorizado por el director de la Revista Conciencia Digital.
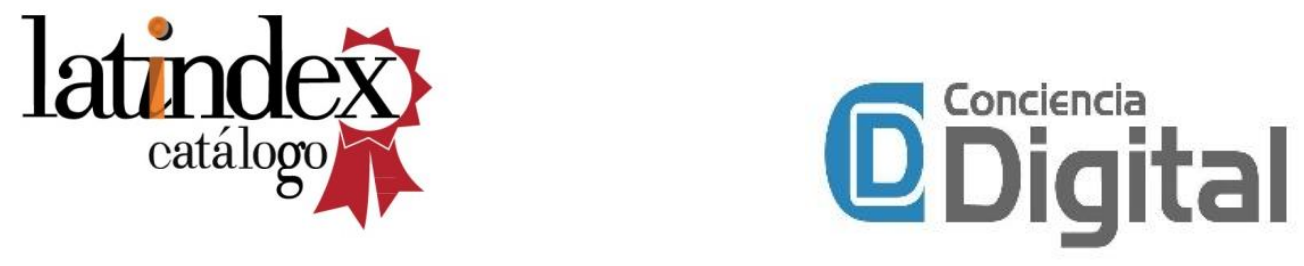